\title{
Risk Assessment in Venous ThromboEmbolism
}

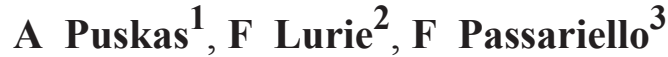

${ }^{1}$ Angio Center-Vascular Medicine, Targu Mures, Romania

${ }^{2}$ Toledo, Ohio (MI, USA). Adjunct Research Professor, University of Michigan. President at American Venous Forum Foundation.

${ }^{3}$ Fondazione Vasculab ONLUS, via Francesco Cilea 280 - 80127 Naples, Italy

The official report of the VTE_Risk Vasculab Debate, held on the Internet, Vasculab List, on Jan 10-31, 2016 (unmoderated session: Feb 1-10, 2016. Discussant: Fedor Lurie, Moderator: Fausto Passariello. submitted: Feb 5, 2017, accepted: Feb 19, 2017, EPub Ahead of Print: Apr 3, 2017, published: May 1, 2017

Conflict of interest: None

DOI: 10.24019/jtavr.12 - Corresponding author: Dr. Fausto Passariello, afunzionale@tiscalinet.it

(C) 2016 Fondazione Vasculab impresa sociale ONLUS. All rights reserved.

Abstract Clinical pre-test probability scores are used to guide the subsequent steps in the diagnosis and therapy of venous thromboembolism. However, often there is resistance in accepting formal and standardized procedures as a substitute of expert judgements. A review of the available tools as well as of the laboratory and imaging techniques is presented, together with the detailed report of a comprehensive debate held on the Vasculab Mailing List. Several unusual topics came to attention during the discussion: as the validation of scores, limited to a few contexts and populations; the over-utilization of venous duplex ultrasound and the rational use of resources; how to choose the steps, managing the amount of time waiting for the results of a test; the scarce attention given today to the long term complications, like the chronic pulmonary embolism, the pulmonary hypertension and the postthrombotic syndrome. A lot of open problems of course remains, the report being witness of the value of a free style atypical discussion, as generally occurs on Vasculab.
Keywords Venous thromboembolism, pre-test probability scores, duplex ultrasound, D-Dimer, sPselectin, chronic thromboembolic pulmonary hypertension.

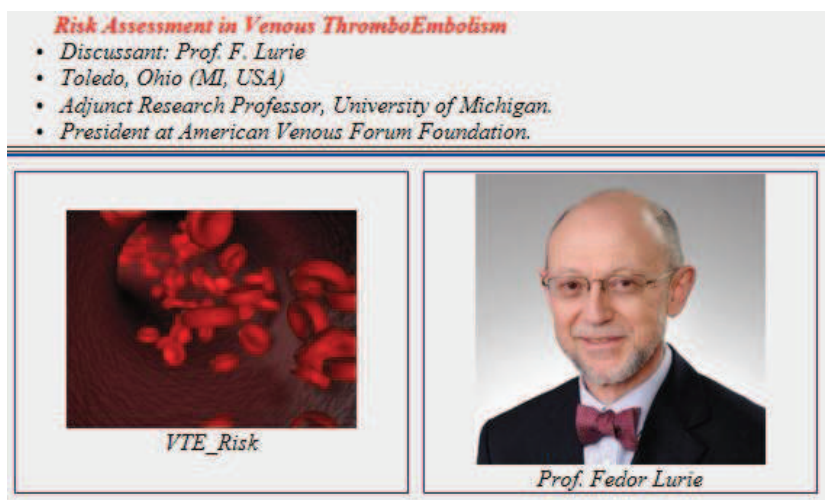

Figure 1 - The VTE_Risk webpage.

DOI: 10.24019/2016.VTE_Risk

\section{Introduction}

Venous thromboembolism (VTE) has gained an increased attention in the last years, owing to our better diagnostic capability and to the availability of new therapeutic choices. Most used acronyms are listed to insure a better comprehension (Table I).

The most common symptom of calf pain has been reported to have sensitivity between $75 \%$ and $91 \%$, and specificity between $3 \%$ and $87 \%$. The reported sensitivity of calf swelling for Deep Veins Thrombosis (DVT) diagnosis ranges from $35 \%$ to $97 \%$, and its reported specificity ranges from $8 \%$ to $88 \%$. In part, such variability is due to the high prevalence of the same signs and symptoms in patients without DVT ${ }^{1,2}$.

Swelling and generally signs of lower limb thrombosis are not the main clinical presentation of VTE, which instead can present itself as a chest pain or a primary 
respiratory failure with hypoxia, which can or cannot be associated to a lower limb thrombosis.

Interestingly, the idea of using risk assessment as the first step in the diagnosis of VTE was developed and promulgated when general consensus was that clinical presentation is unreliable.

The approach was initially developed for symptomatic outpatients, and most frequently used pretest probability (PTP) instruments are validated only in restricted environments and their application remains uncertain for other patient populations, such as pregnant and post-partum women. Instruments that are designed for in-patients are designed for prevention, not for diagnosis of VTE. In addition, between inpatients the greater frequency of DVT is given by adverse effects in medical patients (like for instance post ictus and myocardial infarction), greater than the rate of the well-known VTE in post-operative period (especially in orthopaedic and gynaecologic surgery).

The incidence of VTE changes according to the clinical environment where the patient comes to our observation. Outpatients have a different prevalence of DVT and pulmonary embolism (PE), compared to inpatients and to emergency patients.

Occurrence of provoked VTE now encompasses clinical scenarios that we did not have 20 years ago. For example, the shift is towards seeing more VTE in intensive care units, where the average stay has increased and patients are maintained on life support longer. Unprovoked VTE on the other hand surely is seen more than before probably due to better access to ultrasound. We are probably at a point where studies on current VTE incidences may have to be repeated.

These facts, some of them reported only in a qualitative way, constitute a stimulus to look at VTE as an interdisciplinary pathology, which changes its presentation as soon as you change your point of view, i.e. your working environment.

Applicability of the entire approach as well as of the adopted instruments to other patient populations remains questionable.

\section{The components of diagnosis}

Though the availability of many clinical signs for DVT-PE, the expert-based diagnosis of VTE is considered unreliable ${ }^{3,4}$. Underdiagnosed cases can erroneously attach a DVT-PE label to a patient and be the premise of a low quality prevention and treatment of any future thromboembolic adverse effect.
PTP tools, laboratory assays and imaging techniques are the main components of diagnostic VTE algorithms.

\begin{tabular}{|ll|}
\hline \multicolumn{2}{|c|}{ Acronyms in use in VTE disease } \\
CTEPH & Chronic Thrombo-Embolic Pulmonary \\
CUS & Hypertension \\
DUS & Compression UltraSound \\
ER & Duplex UltraSound \\
HASBLED & Emergency Room \\
& or Liver, (S)troke history, (B)leeding \\
MDCT & Multi Detector Computed Tomography \\
NICE & The National Institute for Health and \\
PERC Rule & Pulmonary Embolism Rule-out Criterion \\
PESI & Pulmonary Embolism Severity Score \\
SPESI & Simplified PESI \\
PIOPED & prospective investigation of the \\
TAPSE & pulmonary embolism diagnosis \\
TTE & Tricuspid Annular Plane Systolic \\
TEE & Excursion \\
V/Q scan & Trans-Thoracic Echocardiography \\
VTE & Trans-Esophageal Echocardiography \\
Table I - Glossary. & Venous Thrombo-Embolism \\
\hline
\end{tabular}

\section{PTP tools}

VTE risk is dependent on the duration of inactivity: induced (anesthesia, surgery) or forced (coma, life-support, paresis, life-threatening illnesses and so on). Most risk assessment scores now take this into consideration.

Several scores are available for VTE (Table II). In order not to ingenerate any confusion, it is worth to describe them shortly.

The most used tools for the assessment of pre-test probability (PTP) in venous thromboembolism (VTE) are: Wells DVT score ${ }^{5,6}$ (Table III), Wells PE score ${ }^{7}$ (Table IV), Revised Geneva score ${ }^{8}$ (Table V) and the pulmonary embolism rule-out criteria (PERC rule) $)^{9,10,11,12}$ (Table VI).

Other scores are not for diagnosis. The Caprini ${ }^{13}$ and Padua ${ }^{14}$ (Table VII) scores quantify the risk of thromboembolism and are useful only for VTE prevention among in-patients. The PESI score ${ }^{15}$ (Table VIII) and its simplified form sPESI score (Table IX) stratify the risk of outcome and death at 30 days in patients suffering for a 
PE episode. The HASBLED score ${ }^{16}$ (Table X) quantifies instead the risk of bleeding, when the patient undergoes oral anticoagulation.

The idea of risk stratification (or pre-test probability) in Wells DVT score was based solely on the population of symptomatic ambulatory patients. Wells PE Score, Geneva and Revised Geneva scores were designed instead for the emergency room (ER).

\begin{tabular}{|lll|}
\hline & \multicolumn{2}{c|}{ PTP Tools } \\
Scores & Environment & Use \\
Wells DVT & outpatients & stratify DVT risk \\
Wells PE & emergency room & stratify DVT risk \\
revised Geneva & emergency room & stratify DVT risk \\
PERC & emergency room & exclude pulmonary \\
& & embolism \\
Padua & inpatients & stratify DVT risk \\
Caprini & inpatients & stratify DVT risk \\
PESI & inpatients & stratify outcome \\
& & risk in pulmonary \\
& & embolism at 30 \\
& & days \\
stratify outcome \\
SPESI & inpatients & risk in pulmonary \\
& & embolism at 30 \\
& & days \\
stratify \\
HASBLED & any & bleeding risk in \\
& & anticoagulated \\
& & patients \\
& & stratify risk in \\
& & VTE patients for \\
& & post-thrombotic \\
& & syndrome \\
& &
\end{tabular}

Table II - List of the most known scores, their working environment and notes on their use

However, apart of a paper about Wells DVT score in the elderly ${ }^{17}$, attempts to do the same for other populations (pregnant and post-partum, cancer, recent trauma or surgery, obesity etc) either failed or have not been suggested.

It is worth reminding once and forever that the postoperative period is not the main cause of VTE, which is much more frequent in the medical department than in the surgical one.

Some of the listed tools are generally not well known by physicians and are also used in an inappropriate way. Indeed, scores used alone have generally no diagnostic value. They serve instead to classify the patient into risk classes. Subsequent diagnostic choices depend on the selected class.

\begin{tabular}{|c|c|}
\hline \multicolumn{2}{|l|}{ The Wells DVT score } \\
\hline Clinical characteristic & Score \\
\hline $\begin{array}{l}\text { Active cancer, (patient receiving treatment for } \\
\text { cancer within the previous } 6 \text { months or currently } \\
\text { receiving palliative treatment) }\end{array}$ & 1 \\
\hline $\begin{array}{l}\text { Paralysis, paresis, or recent plaster } \\
\text { immobilization of the lower extremities }\end{array}$ & 1 \\
\hline $\begin{array}{l}\text { Recently bedridden for } 3 \text { days or more, or major } \\
\text { surgery, within the previous } 12 \text { weeks requiring } \\
\text { general or regional anesthesia }\end{array}$ & 1 \\
\hline $\begin{array}{l}\text { Localized tenderness along the distribution of } \\
\text { the deep venous system }\end{array}$ & 1 \\
\hline Entire leg swollen & 1 \\
\hline $\begin{array}{l}\text { Calf swelling at least } 3 \mathrm{~cm} \text { larger than that on } \\
\text { the asymptomatic side (measured } 10 \mathrm{~cm} \text { below } \\
\text { tibial tuberosity) }\end{array}$ & 1 \\
\hline Pitting edema confined to the symptomatic leg & 1 \\
\hline Collateral superficial veins (nonvaricose) & 1 \\
\hline Previously documented DVT & 1 \\
\hline Alternative diagnosis at least as likely as DVT & -2 \\
\hline $\begin{array}{l}\text { Table III - The Wells DVT score is used for out-pat } \\
\text { the Ambulatory for the quick computation of the pr } \\
\text { probability for DVT, as a hint to suggest humoral } \\
\text { instrumental investigations. }\end{array}$ & $\begin{array}{l}\text { ents in } \\
\text {-test } \\
\text { nd/or }\end{array}$ \\
\hline
\end{tabular}

Wells DVT Score is designed and validated to be used only in ambulatory outpatients. When VTE is suspected at the visit, the score is computed: if low, ask for a D-Dimer assay; if otherwise it is high, prescribe a Duplex UltraSound (DUS). The patient goes home and comes back (if not lost), generally in 7-15 days with the results. The physician waits a so long time for a diagnostic assessment and only the ambulatory environment can justify it, because the patient generally has no urgent symptom. The tree-like hierarchy of the algorithm can be explained only by the ambulatory environment. Wells DVT Score cannot be applied and is not validated in the ER, where a D-Dimer can be obtained in 1-2 hours following traditional laboratory methods, but also in 5'-20' using specialised bedside assays which are not so rare nowadays.

ERs use instead the Geneva ${ }^{18}$ or the Wells PE score for people suspected for an important VTE, which can have life-threatening consequences. In addition, the PERC rule (Pulmonary Embolism Rule-out Criteria) is often used, which has the advantage of being simple and quick. If all the listed items in PERC are satisfied, pulmonary embolism is ruled-out. In addition, the efficiency of the PERC rule can be improved introducing also the Gestalt, i.e. the personal intuition of the operator about the presence/absence of PE. 
Thus personal feelings together with a satisfied PERC Rule are very effective to exclude PE (not DVT) in the ER.

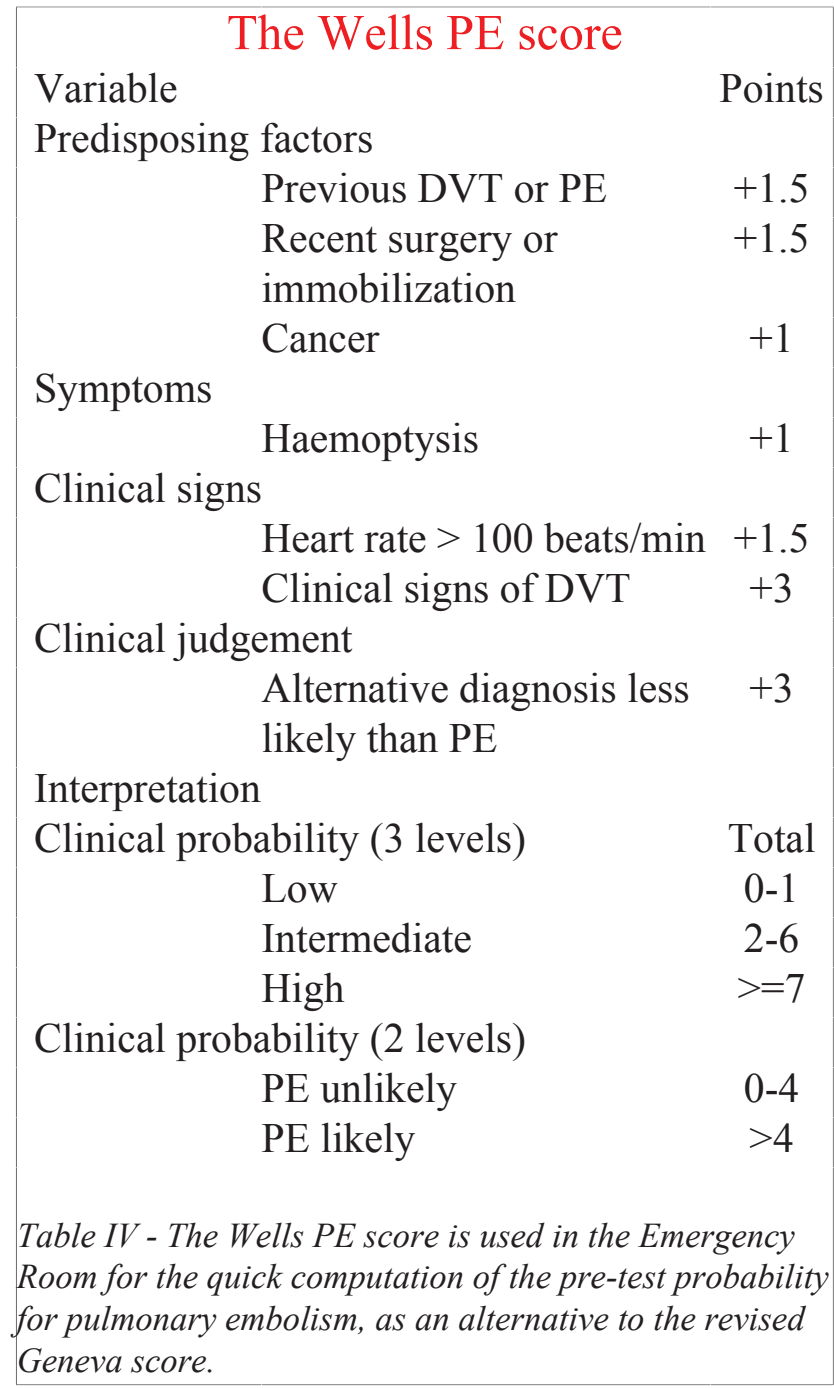

\section{Discussion}

Very often it is not possible to find the embolism origin in PE: whether you use DUS, CT or MRI it is not important, very often PE seems isolated (F Passariello, M Patel). The origin can be elsewhere than in lower legs (everywhere in oncologic and rheumatic patients, right ventricle infarct, pelvis veins, and so on). Even in the legs, thrombi can hide in the calf and it can be difficult to detect them.

There is a great resistance among physicians to adopt PTP scores (the best known PTP score in this discussion is Wells DVT score but it is rarely utilized). Some people in the debate consider scores only as simple tools for family practitioners (A Pieri), whilst specialists' in the field can practically ignore them, owing to their competence (D Casian, Z Lazarashvili). This point of view is misleading, as scores were effectively introduced as tools to bypass the uncertainty of the clinical diagnosis and to avoid the so called Expert Opinion, which is non-standardisable and not useful for comparisons.

Other physicians participating in the debate recognize the clinical value of scores, but prefer to be non-methodical in their practical use, i.e. they don't use written forms, but declare to compute the scores mentally (H Schlup). This is questionable, because nothing guarantees that the mental procedure was effectively complete, as its details are not shared.

An extreme position in this debate is to assign a great value to DUS as screening investigation, i.e. applied to every people suspected for VTE (D Casian, A Puskas, H Schlup, PL Antignani, A Pieri, Z Lazarashvili). This seems an example of bad use of resources. Imagine what could happen in a high number access hospital with a small number of DUS specialists'. Even in private practice this is not an appropriate behaviour, as it increases costs. Cost is not only money, but also time to wait for the examination and time and the amount of work to perform it.(F Passariello, G Stansby, M Schul)

Another respectable position is to use a low PTP threshold to ask for a DUS or to perform it directly in the ER. Well, if the DUS expert is available (or if you are the DUS expert), this behaviour is comprehensible, though it cannot be shared. Consider the case when you have a lot of concurrent requests in your work and you have to assign a priority. For instance, 10 patients need a DUS evaluation for a suspected VTE, but they have different PTP scores. Will you perform the DUS without any order or will you satisfy the requests according to their priority? This is the meaning of a rational use of resources, which becomes more and more important as long as ER services become more widely spread.

The problem of over-utilization of DUS and other resources is well-known ${ }^{19}$ and we will come back to it later. At the moment, it is useful to anticipate that the appropriate use of diagnostic resources must be guided by a reliable algorithm.

\section{Laboratory assays}

Laboratory assays are useful as the clinical signs and symptoms of VTE are nonspecific, the accuracy of clinical diagnosis being less than $50 \%$. It would be useful to use biomarkers that enable early identification of patients at high or low risk of primary and recurrent VTE. Several established and new biomarkers associated with VTE have been investigated with regard to their potential for predicting primary or recurrent VTE, for facilitating the diagnosis and for optimizing the clinical management of VTE.

\section{D-Dimer}

D-Dimer ${ }^{20,21,22}$ is used as an initial screening test in the ER to diagnose patients who have signs, or symptoms suggestive of VTE. Before ordering the D-dimer test, it is essential to assess the patient's clinical probability for VTE, for instance by means of the Wells score. D-dimers are degradation products formed when cross linked fibrin contained within a thrombus is proteolyzed by plasmin. D-dimers may be produced in many diseases and conditions associated with thrombosis and thrombolysis. Thus, although presence of D-dimers is specific for degradation of cross-linked fibrin, cross-linked fibrin is not specific for VTE. Being ubiquitous, D-dimer is not generally useful in confirming a diagnosis of VTE.

Clinicians must be aware that D-dimer is increased in many conditions. Physiologic causes of D-dimer elevation include pregnancy and puerperium, increasing age ( $>65$ years), Afro-American heritage, cigarette smoking, recent trauma, and the postoperative period. Pathological causes for elevated D-dimer levels are: VTE, arterial thrombosis, atrial fibrillation, liver disease, renal disease, infections, malignancy and its treatment, chronic inflammatory disease, etc. HighPTP patients should not be tested for D-dimer because the post-test probability for a D-dimer $>500 \mu \mathrm{g}$ FEU/1 has a poor positive predictive 
value for VTE. However, a negative D-dimer is useful in ruling out VTE in low-PTP patients. The cutoff threshold may differ over age groups, for instance in the elderly an increased value of $>750 \mu \mathrm{gEU} / 1$ was proposed. Many factors play a key role in changing the sensitivity and specificity of D-dimer testing, including the extent of thrombosis and fibrinolytic activity, duration of symptoms, anticoagulant therapy, comorbidity due to surgical or medical illnesses, inflammatory diseases, cancer, elderly age, pregnancy and the postpartum period, and previous VTE.

Many studies have shown that the D-dimer test is highly sensitive $(>95 \%)$ in acute deep venous thrombosis or pulmonary embolism, usually with a cut-off value of $500 \mu \mathrm{g}$ FEU/1, which reasonably rules out acute VTE in low-PTP patients. Patients with high D-dimer levels upon presentation may prompt a more intense diagnostic approach, irrespective of pretest probability. Studies performed after a negative $\mathrm{D}$-dimer for 3 months proved the high negative predictive value (NPV) of D-dimer testing in low-PTP patients with suspected VTE. The combination of the D-Dimer and Wells criteria can in significantly reduce the number of patients referred for venous Duplex ultrasound. In unselected inpatients, D-dimer testing has limited clinical utility because of its poor specificity. D-dimers are detected by immunoassays using monoclonal antibodies specific for the crosslinked D-dimer domain in fibrinogen. Commercially available assays include latex agglutination, immunoturbidimetry, and enzyme-linked immunosorbent assay (ELISA). Efforts made to standardize D-dimer results have not been successful thus far, because the D-dimer analyte is not uniform across the different assays ${ }^{23}$.

\section{sP-selectin}

Soluble P-selectin ${ }^{24,25}$ (sP-selectin) is a member of the selectin family of cell adhesion molecules and is primarily stored in the alpha granules of platelets and the Weibel-Palade bodies of endothelial cells. After activation of platelets and endothelial cells, P-selectin is translocated to the cell surface and in part released into the plasma in soluble form. P-selectin acts through binding to its main counterreceptor, the P-selectin glycoprotein ligand-1 (PSGL-1), located on leukocytes. P-selectin is an important molecule in hemostasis and thrombosis. The P-selectin receptor, PSGL-1, is also expressed on platelets and mediates platelet-endothelium interaction and supports fibrin formation and thrombus growth. The interaction of P-selectin and its receptor leads to several mechanisms that induce a pro-coagulant state. Studies have demonstrated the clinical significance of P-selectin for thrombosis, and elevated sP-selectin has been implicated as a risk factor for venous thromboembolism. sP-selectin levels have been observed to increase during an acute event of VTE. There is growing evidence that platelet activation results in higher levels of sP-selectin, and it can be assumed that $\mathrm{SP}$-selectin is mainly platelet-derived and reflects platelet activation.

Plasma levels of sP-selectin are elevated in acute DVT. Further, high levels of sP-selectin were recently associated with an increased risk for recurrence of DVT and in cancer patients, high plasma levels of sP-selectin were predictive of VTE. In one study soluble sP-selectin in combination with the Wells score, established the diagnosis of DVT in lower limbs with a specificity of $96 \%$ and a positive predictive value of $100 \%$. Another recent study showed that when Wells score $>2$, sPselectin is an excellent biomarker to rule in DVT in lower limbs. Ddimer and a Wells score $<2$ was most sensitive at excluding a diagnosis of DVT. However the clinical applicability of sP-selectin measurements to assess the risk of VTE needs to be standardized and investigated in greater extent in interventional trials.

\section{Oximetry and Acid Base Balance}

Essentially, PE affects the oxygen transport and changes can be detected by means of the following measurements: oximetry, with detection of low peripheral oxygen saturation $\left(\mathrm{SpO}_{2}\right)$ and Acid Base Balance (ABB). As regards the $\mathrm{ABB}$, there are 3 parameters:

\section{ABB parameters and $\mathrm{PE}$}

- $\quad$ 1. Hypoxia (low arterial $\mathrm{pO}_{2}$ ) and hypocapnia (low arterial $\mathrm{CO}_{2}$ );

- 2. Increased alveolar(A)-arteriolar(a) difference( $\Delta$ ) of oxygen $\left(\Delta_{\mathrm{A}-\mathrm{a}} \mathrm{O}_{2}\right)$

3. Increased ventilation/perfusion ratio $\left(\frac{\mathrm{V}}{\mathrm{Q}}\right)$.

The first two ABB parameters can be measured with in an arterial/ capillary sample, whilst the third one requires an arterial and a central venous sample ${ }^{26-28}$. Though very informative of the severity of pulmonary embolism, $\mathrm{AAB}$ parameters are not included in any officially recognized diagnostic algorithm for PE. Apart of measurement errors, a general relationship should generally hold:

$$
\mathrm{S}_{\mathrm{a}} \mathrm{O}_{2}>=\mathrm{S}_{\mathrm{p}} \mathrm{O}_{2}>=\mathrm{S}_{\mathrm{V}} \mathrm{O}_{2}
$$

where $\mathrm{SO}_{2}$ is the oxygen $\%$ hemoglobin saturation and the subscripts $\mathrm{a}, \mathrm{p}$ and $\mathrm{v}$ mean arterial, peripheral and venous respectively.

\begin{tabular}{|c|c|c|}
\hline \multicolumn{3}{|c|}{ The revised Geneva score } \\
\hline Variable & & Points \\
\hline \multicolumn{3}{|c|}{ Predisposing factors } \\
\hline & Age $>65$ years & +1 \\
\hline & Previous DVT or PE & +3 \\
\hline & Surgery or fracture within & +2 \\
\hline & 1 month & \\
\hline & Active malignancy & +2 \\
\hline \multicolumn{3}{|l|}{ Symptoms } \\
\hline & Unilateral lower limb pain & +3 \\
\hline & Haemoptysis & +2 \\
\hline \multicolumn{3}{|c|}{ Clinical signs } \\
\hline \multicolumn{3}{|c|}{ Heart rate } \\
\hline & $75-94$ beats/min & +3 \\
\hline & $>=95$ beats $/ \mathrm{min}$ & +5 \\
\hline & $\begin{array}{l}\text { Pain on lower limb deep } \\
\text { vein at palpation and } \\
\text { unilateral oedema }\end{array}$ & +4 \\
\hline \multicolumn{3}{|c|}{ Interpretation } \\
\hline & Clinical probability & Total \\
\hline & Low & $0-3$ \\
\hline & Intermediate & $4-10$ \\
\hline & High & $>=11$ \\
\hline \multicolumn{3}{|c|}{$\begin{array}{l}\text { Table V-The revised Geneva score is used in the } \\
\text { Emergency Room for the quick computation of the pre-test } \\
\text { probability for pulmonary embolism, as an alternative to } \\
\text { the Wells PE score. }\end{array}$} \\
\hline
\end{tabular}




\section{Discussion}

F Lurie states that D-dimer is the most frequently used test and many aspects of its utilization are well-known. The issues are a) variability in the diagnostic properties among different assays, b) low positive predictive value, and c) an established cut-off is missing in some populations of patients (pregnant and post-partum, post-surgical, trauma etc.). On the contrary, there is a general consensus that the sensitivity of D-dimer is high, though in literature it ranges from $60 \%$ to $96 \%$. Similar to clinical evaluation, use of D-dimer as a single diagnostic tool may result in inadequate management of more than $15 \%$ of patients with suspected DVT. A Puskas states that the D-dimer is useful (good sensitivity but low specificity) to rule out DVT only when the Wells probability score is low. G Stansby's opinion is that we don't have to consider D-dimer separately, on the contrary what is required is an algorithm that incorporates a clinical prediction score, D-dimer and imaging. As such D-dimer would usually precede any other decision about ultrasound investigations. F Passariello argues that the reported use of D-Dimer together with the Wells score was validated only in an ambulatory setting. M Patel considers $G$ Stanby's opinion most valid which means clinical scoring, laboratory test and imaging all in combination in case imaging creates a doubt. M Boucelma uses also D-dimer as a help in taking decisions when the clinical probability is mild and no thrombosis is evident in US and or scanner, she writes.

F Passariello argues that D-Dimer has no useful value in highPTP patients. In synthesis, he tells that D-Dimer must be used as a rule-out criterion ONLY in low risk patients. Special bedside D-dimer assays are not so rare nowadays making possible their use in the ER. $M$ Patel summarizes that the most reliable way of detecting DVT is based on 1. Clinical scoring 2. Duplex scanning with or without CT pulmonary angio 3. D-dimer test. F Passariello would like also to add CUS and SP-selectin to be included into a diagnostic algorithms for VTE. M Boucelma's opinion is that concentration of P-selectin and D-dimer after acute DVT may be a predictor of recurrent venous thromboembolism, and P-selectin inhibition promotes resolution better than enoxaparin. This biomarker may be included in thromboembolism algorithm, though almost all laboratories are not organized to measure it, replies $\boldsymbol{F}$ Passariello. He states that $s P$-selectin is validated to confirm VTE in high risk (high pretest score) patients, while it has a limited value in low risk ones. $\boldsymbol{S}$ Szarka's algorithm starts from Ddimer. In his practice all patients suspected for VTE receive a D-Dimer test and then based on clinical pre-test probability the patient may or may not get an ultrasound examination. H Schlup informs us that the price of a D-dimer test is $25 \$$ in Brasil and it takes 3 days for the result while a DUS costs $45 \$$ and is quickly available. After the DUS we probably don't need D-dimer but after D-dimer we probably will ask for DUS. In private practice usually the doctor doesn't have time to wait, he argues, thus the question arises why not asking directly for DUS.

In conclusion almost all discussants agreed with the utility of biomarkers, especially of D-dimer in the diagnostic algorithm of VTE in combination with a clinical probability score and an imaging method (ultrasound and/or CTPA).

\section{Available imaging methods}

\section{Ultrasound}

\section{TTE}

Trans-Thoracic Echocardiography (TTE) can add useful information. Right heart chambers dimensions can increase considerably their diameters in acute PE. The pulmonary artery tension can be non-invasively estimated from right chambers velocimetry and inferior vena cava caliber and pulsatility. In addition, the Tricuspid Annular Plane Systolic Excursion (TAPSE) can evaluate the diastolic function of right heart chambers. These observation can help in assessing patients with $\mathrm{PE}$ or chronic thromboembolic pulmonary hypertension (CTEPH) and can match the corresponding changes in the electrocardiogram, which however are more reliable in chronic pulmonary hypertension (CPH).

\section{DUS}

Duplex ultrasonography (DUS) is a recognized ultrasound diagnostic tool in vascular diseases and of course in venous pathology. A training period (at least 2 years) is required to prepare a skilled ultrasonographer. Owing to its complexity DUS cannot be described here, thus we refer to any available educational text.

\section{The pulmonary embolism rule-out criteria (PERC rule)}

Age $<50$ years

Pulse $<100 \mathrm{bpm}$

Pulse oximetry $>94 \%$

No unilateral leg swelling

No hemoptysis

No surgery or trauma within 4 weeks

No prior DVT or PE

No oral hormone use

Table VI - The pulmonary embolism rule-out criteria (PERC rule) is used in the Emergency Room for the quick exclusion of pulmonary embolism. It works better when associated to the personal feelings of the operator (Gestalt).

\section{CUS}

Compression ultrasonography (CUS) may be proficiently learned in $<2$ hours. It was developed as a quick and simple emergency tool, in order to give reliable results in cases of suspected $\mathrm{DVT}^{29-33}$. It requires only a simple BMode scan of groin and popliteal region (2points ultrasonography), where observation can be extended to the 3rd order confluence of anterior and posterior tibia and peroneal veins. This extension however is debated, because it is a more difficult manoeuvre which requires a longer training period.

Simple BMode scanning is used to look at the saphenousfemoral junction (SFJ) and saphenous-popliteal junction (SPJ), which can just visualize the thrombus inside the lumen, so providing the diagnosis.

A soft compression of the cited veins with the echo probe can show the flexible state of their wall. When the vein is compressible, CUS is negative, when the vein is rigid CUS is positive. In almost all cases compressibility implies patency, while rigidity implies occlusion. In several cases, it's possible to get intermediate results, i.e. partially compressible, which means partial thrombosis or recanalization of a vein.

There are false positive cases, due to tissue sclerosis, because veins are inside a not easy compressible compartment. However, this does not occur in lymph diseases, because generally veins remain compressible even if inside a heavy altered tissue compartment. There 
are also false negative cases (thrombosis is elsewhere, i.e thrombus localized above or below the junctions and therefore non affecting junctions compressibility).

\begin{tabular}{|c|c|}
\hline \multicolumn{2}{|l|}{ The Padua score } \\
\hline Baseline features & Score \\
\hline Active cancer* & 3 \\
\hline $\begin{array}{l}\text { Previous VTE (with the exclusion of } \\
\text { superficial vein thrombosis) }\end{array}$ & 3 \\
\hline Reduced mobility $^{\dagger}$ & 3 \\
\hline $\begin{array}{l}\text { Already known thrombophilic } \\
\text { condition }\end{array}$ & 3 \\
\hline $\begin{array}{l}\text { Recent ( }>=1 \text { month) trauma and/or } \\
\text { surgery }\end{array}$ & 2 \\
\hline Elderly age $(>=70$ years $)$ & 1 \\
\hline Heart and/or respiratory failure & 1 \\
\hline $\begin{array}{l}\text { Acute myocardial infarction or ischemic } \\
\text { stroke }\end{array}$ & 1 \\
\hline $\begin{array}{l}\text { Acute infection and/or rheumatologic } \\
\text { disorder }\end{array}$ & 1 \\
\hline Obesity $(\mathrm{BMI}>=30)$ & 1 \\
\hline Ongoing hormonal treatment & 1 \\
\hline $\begin{array}{l}\text { Table VII - The Padua score is used for the assessn } \\
\text { VTE risk in in-patients. } \\
\text { Risk assessment model (high risk of VTE: }>=4 \text { ) } \\
\text { *Patients with local or distant metastases and/or i } \\
\text { chemotherapy or radiotherapy had been performed } \\
\text { previous } 6 \text { months. }\end{array}$ & $\begin{array}{l}\text { ment of } \\
\text { in whom } \\
\text { din the }\end{array}$ \\
\hline $\begin{array}{l}\text { Hedrest with bathroom privileges (either due to } p \\
\text { limitations or on physicians order) for at least } 3 d c\end{array}$ & $\begin{array}{l}\text { atient's } \\
\text { lays. }\end{array}$ \\
\hline $\begin{array}{l}\text { Carriage of defects of antithrombin, protein C or } \\
\text { S, factor V Leiden, G20210A prothrombin mutatio } \\
\text { antiphospholipid syndrome. }\end{array}$ & \\
\hline
\end{tabular}

Other difficult cases are the absence of the greater saphenous vein or of the shorter saphenous vein, as normal anatomy is changed.

CUS is a valid tool in proximal lower limb symptomatic DVT, with a sensitivity of $97 \%$ and specificity of $98 \%$. In lower limb distal DVT results are conflicting.

When the pre-test probability is high or the clinical suspicion is important and CUS is negative, it is possible to use a second CUS at 1 week. This second look or serial CUS can be used also to monitor the patient (when symptoms persist or increase) or in the post-operative period in patient at high risk.

CUS is a separate exam, not a DUS, though this latter includes compression on veins. In addition, DUS can visualize the echogenicity of the venous thrombus, its adherence to the venous wall and its mobility inside the lumen (floating thrombus). DUS looks also at flow (absent / present) and at its changes during breathing and functional manoeuvres.
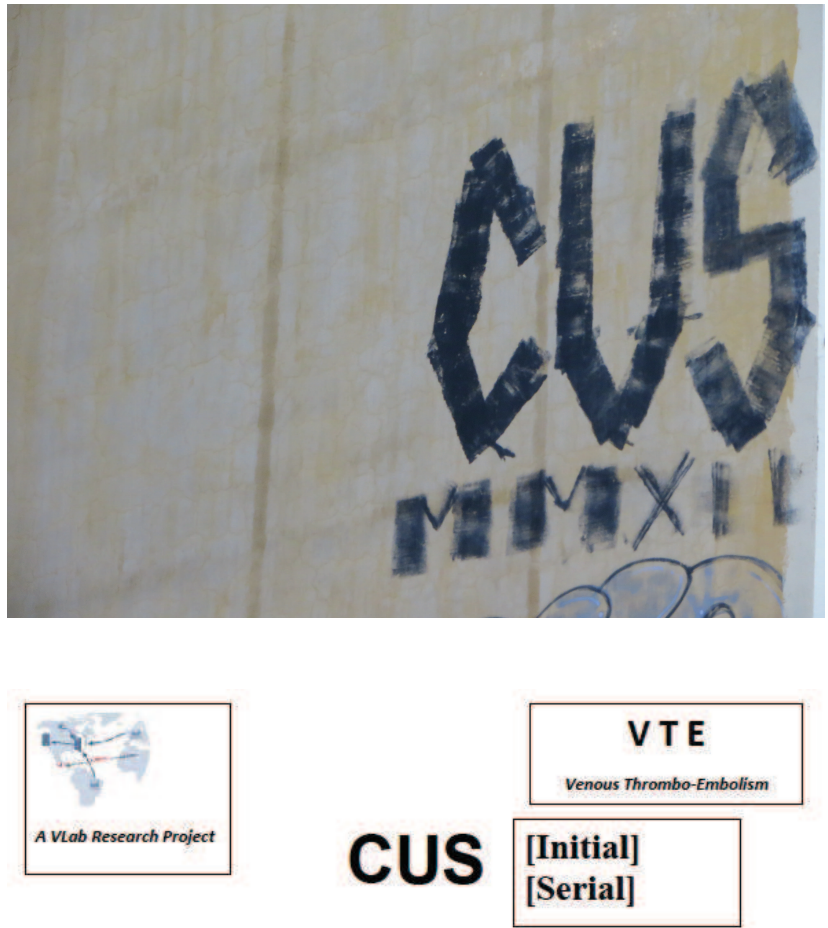

Patient

$[\mathrm{M}][\mathrm{F}]$ age N. Ref. [ER] [Clin dossier] [other]

\begin{tabular}{|c|l|l|}
\hline CUS & \multicolumn{1}{|c|}{ RT } & \multicolumn{1}{c|}{ LT } \\
\hline \multirow{3}{*}{ groin } & $\begin{array}{l}{[-][+]} \\
\text { Note } \\
\text { res }\end{array}$ & $\begin{array}{l}{[-][+]} \\
\text { Note } \\
\text { popliteal }\end{array}$ \\
\hline & $\begin{array}{l}{[-1[+]} \\
\text { Note }\end{array}$ & \begin{tabular}{l}
{$[-][+]$} \\
Note \\
\hline
\end{tabular} \\
\hline
\end{tabular}

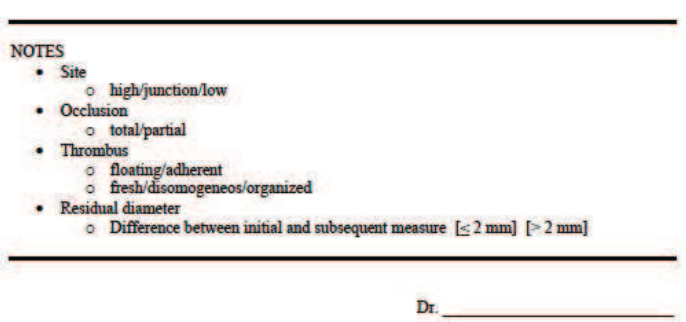

Figure 2 - The VLab CUS form for the Emergency Room

Patients with suspected DVT of the lower limbs are usually investigated with CUS or DUS. The latter approach has a much greater diagnostic capability, as for instance its ability to detect isolated calf vein thrombosis. However, it requires skilled operators and depends on the availability of an experienced ultra-sonographer mainly during the ordinary working hours. A prospective, randomized, multicenter study of consecutive symptomatic outpatients $(n=2465)$ with a first episode of suspected DVT of the lower extremities shows that 2 diagnostic strategies (1. CUS+D-dimer when CUS is normal, 2. whole length DUS) are equivalent when used for the management of symptomatic 
outpatients with suspected DVT of the lower extremities. Relevant features of this strategy are simplicity, reproducibility, and broad availability. There is the need to repeat the test within 1 week (serial CUS) in patients with normal findings at presentation and positivity of D-dimer. With DUS strategy color flow is exploited to enhance small vessel visualization, although vein compressibility still constitutes the main diagnostic criterion. The advantage of the DUS approach is the ability to exclude isolated calf DVT, allowing for 1-day low molecular weight heparin (LMWH) treatment of all patients, eventually stopping the therapy in case of negative DUS without any additional testing. Conversely, it needs top-quality ultrasound equipment and experienced operators; therefore, it is often unavailable after hours and during the weekends.

\section{Other imaging techniques}

The diagnosis of pulmonary embolism (PE) remains a common challenge confronting physicians in daily clinical practice. PE is considered in the differential diagnosis of many clinical presentations and in a wide variety of clinical settings. Ventilation-perfusion lung scanning (V/Q) has been the non-invasive imaging procedure of choice in patients with suspected PE for many years. However, a majority of patients with suspected PE undergoing a ventilation-perfusion scan have a non-diagnostic examination (low or intermediate probability of $\mathrm{PE}$ ). The prospective investigation of the pulmonary embolism diagnosis $^{34,35}$ (PIOPED) study shows that clinical assessment combined with the ventilation/perfusion scan established the diagnosis or exclusion of pulmonary embolism only for a minority of patientsthose with clear and concordant clinical and ventilation/perfusion scan findings. In PIOPED era the gold standard was pulmonary angiography. More recently, the computed tomographic pulmonary angiography (CTPA) has been introduced as an alternative non-invasive test. A CTPA provides a clear result (either positive or negative for PE) and possibly an alternative diagnosis to explain the patient's symptoms. Multiple-detectors CTPA have a higher sensitivity for PE as compared with single-detector CTPA. In particular, multiple-detectors CTPA allows better visualization of segmental and subsegmental pulmonary arteries. However, V/Q scintigraphy could have a similar or even higher sensitivity than CTPA in detecting CTEPH as a potential curable cause of pulmonary hypertension ${ }^{36}$. CTPA is now preferred as the first-choice test for PE by both scientific societies and practicing physicians. The increased sensitivity of CTPA may have a downside: the detection of emboli that are so small as to be clinically insignificant.

This phenomenon has been called "overdiagnosis", defined as the detection of an abnormality that will never cause symptoms or death ${ }^{37,38}$. Overdiagnosis matters because it can lead to iatrogenic harm. While a clinically non-significant $\mathrm{PE}$ is by definition not harmful, treating such an embolism can cause harm (e.g., bleeding from anticoagulation, which can in the worst case be fatal). On the other site of the coin is CTEPH as a consequence of repetitive emboli even if they are small. The judicious balance between these two ends of the spectrum of the disease needs further investigations. For certain patient groups, such as patients with contraindications to iodinated contrast media and young women (possibility of pregnancy) with a low PEPTP, magnetic resonance (MRI) can be considered as a first-choice imaging tool for PE assessment. Recent technical developments have substantially improved the potential of MRI for PE diagnosis ${ }^{39,40}$, as the development of short magnets and dedicated whole-body MRI systems together with the dynamic gadolinium enhancement, which allow a comprehensive evaluation of pulmonary embolism and deep venous thrombosis in a single exam. The introduction of parallel imaging has substantially improved the spatial and temporal resolution of pulmonary MR angiography. By combining timeresolved pulmonary perfusion MRI with high-resolution pulmonary
MRA a sensitivity and specificity of over $90 \%$ is achievable, which is comparable to the accuracy of CTPA ${ }^{41}$.

\begin{tabular}{|c|c|}
\hline \multicolumn{2}{|l|}{ The PESI score } \\
\hline Age & $\begin{array}{c}+ \text { N. of } \\
\text { years }\end{array}$ \\
\hline Sex Male & +10 \\
\hline History of Cancer & +30 \\
\hline Heart Rate $>=110$ & +20 \\
\hline Systolic BP $<100 \mathrm{~mm} \mathrm{Hg}$ & +30 \\
\hline Respiratory Rate $>=30$ & +20 \\
\hline Temperature $<36^{\circ} \mathrm{C} / 96.8^{\circ} \mathrm{F}$ & +20 \\
\hline $\begin{array}{l}\text { Altered Mental Status } \\
\text { (Disorientation, lethargy, stupor, } \\
\text { coma) }\end{array}$ & +60 \\
\hline $\mathrm{O}_{2}$ Saturation $<90 \%$ & +20 \\
\hline $\begin{array}{l}\text { Table VIII - The pulmonary embolism sev } \\
\text { (PESI) is a } 11 \text { items score that predicts th } \\
\text { at } 30 \text { days in pulmonary embolism patien } \\
\text { adds a value to the age of the patient. Acc } \\
\text { total, the risk is considered very low }<=6 \\
\text { intermediate (86-105), high (106-125), ve }\end{array}$ & $\begin{array}{l}\text { ndex } \\
\text { ome } \\
\text { h item } \\
g \text { to the } \\
v(66-85) \text {, } \\
h(>125)\end{array}$ \\
\hline
\end{tabular}

The sPESI score
Age $>80$ years
History of cancer
History of chronic cardiopulmonary disease
Heart rate $>=110$
Systolic BP $<100 \mathrm{~mm} \mathrm{Hg}$
$\mathrm{O}_{2}$ saturation $<90 \%$
Table IX- The simplified PESI (sPESI) score uses a
reduced set of items than PESI to predict the outcome at
30 days in pulmonary embolism patients. Low risk if all
criteria are unsatisfied, high risk even if just one criterion
is satisfied.

\section{Discussion}

$\boldsymbol{F}$ Lurie states that studies increasingly confirm that imaging methods are much less accurate than we think. A Puskas's opinion is that bilateral whole length DUS of the lower extremities (in laying, sitting and sometimes in standing position, including tibialfibulal gastrocnemius/soleus veins) has a very good sensitivity and specificity in DVT diagnosis. We perform it in every suspect patient, he says. It takes about 20 minutes but of course the accuracy is operator dependent and every center needs experienced specialists. In his opinion this specialist has to be a clinician (angiologist/cardiologist) vascular surgeon/internist) rather than a radiologist. $\boldsymbol{M}$ Patel and $\boldsymbol{F}$ Lurie agree that it is not possible to look at all possible sources of emboli, so something is always missed. We all can see that occasional CT angiography of the chest shows PE when there is no DVT seen in the lower extremities. Another issue is that the false positive rate of DUS 
in low risk patients is about $4 \%$, but the actual incidence is $<1 \%$. If you do ultrasound on all patients, and treat all positives, you uselessly treat at least 3 out of 100 patients. Is this an acceptable rate?-raises the question F Lurie. D Casian says that his threshold for DUS is very low. If he has a minimal clinical suspicion of DVT, he performs DUS or at least 2 point CUS (popliteal and common femoral vein). In his institution the decision of the vascular surgeon usually is an empirical one, based on clinical experience. A Puskas emphasizes again that he performs in almost all cases CUS and DUS. This is whole length Duplex including tibia and fibula veins, especially in case of high Wells score. He mentions that his practice is an angiology ambulatory unit and patients come to him usually with leg symptoms. PL Antignani, H Schlup, Z Lazarashvili and G Peruzzi agree all with A Puskas' statement. H Schlup says that the DUS can be done immediately, while the D-dimer in Brasil takes some days. His sonographer is a doctor (not a technician) who does just DUS all the day. Z Lazarashvili states that DUS of the lower limbs is the first option not only for diagnosis of DVT, but also in ER when patient comes with suspicion on PE in combination with echocardiography. Only later it is possible to use other possible tools for diagnosis (D-dimer, CT-angiography, etc.). He thinks it is a right way to prevent repeated embolism.

\begin{tabular}{|ll|}
\hline \multicolumn{2}{|c|}{ The HASBLED score } \\
Hypertension & $\begin{array}{l}\text { Uncontrolled, }>160 \mathrm{mmHg} \\
\text { systolic }\end{array}$ \\
Renal disease & $\begin{array}{l}\text { Dialysis, transplant, } \mathrm{Cr}>2.26 \\
\mathrm{mg} / \mathrm{dL} \text { or }>200 \mu \mathrm{mol} / \mathrm{L}\end{array}$ \\
Liver disease & $\begin{array}{l}\text { Cirrhosis or bilirubin }>2 \mathrm{x} \\
\text { normal with AST/ALT } / \mathrm{AP}>3 \mathrm{x} \\
\text { normal }\end{array}$ \\
Stroke history &
\end{tabular}

\section{Prior major bleeding or predisposition to bleeding}

Labile INR. $\begin{aligned} & \text { Unstable/high INRs, time in } \\ & \text { therapeutic range }<60 \%\end{aligned}$
A

Age $>65$

\section{Medication usage predisposing to bleeding. Antiplatelet agents, NSAIDs}

\section{Alcohol or drug usage history - > $=8$ drinks/week}

Table X - The HASBLED score was introduced to evaluate the risk of bleeding in the treatment of atrial fibrillation. The score can be applied also to the treatment of venous thromboembolism. Each item is valued 1 point and the risk is high for a score $>=3$.

F Passariello points out that DUS and the CUS are different examinations and considering CUS as a simple manoeuvre performed during DUS, increases the confusion. He also states that very often it is not possible to find the embolism origin in PE whether one uses
DUS or CT or MRI. M Patel thinks that over-treating is acceptable if a repeat DUS is carried out as a serial follow-up exam after one week. Anti-coagulation during that time is safe as complications of anti-coagulation are related mostly to duration. If the follow-up DUS is negative then anticoagulation should be withdrawn, he states. $\boldsymbol{G}$ Stansby replies that making DUS for everyone with suspected DVT is not a very resource efficient service. F Passariello completely disagrees with the great value assigned to DUS as a screening investigation, i.e. applied to every people suspected for VTE. This is an example of bad use of resources, he argues. CUS, D-Dimer and DUS must be used after a selection guided by scores. DUS is not CUS and vice versa, he states. Though he is a DUS expert, he must recognize that CUS in the ER is more useful, because it can be performed by a lot of operators, trained for CUS (1 morning), but not for echo Doppler (2 years). Having a lot of exams for a screening, even if not so precise, catches VTE better than a few precise examinations. In Moldavia, says D Casian, DUS with well-trained specialist is available only in large hospitals (usually University or Research Center) and during the working hours. In rural area and small hospitals CUS (usually available 24/24) + clinical scores probably will remain the optimal strategy for DVT diagnosis. In Dr Casian's hospital he investigates suspected DVTs and experiments a low rate of DUS false negative, when the patient is referred by the Vascular Surgeon and not by another specialist.

There are two weak points in this argumentation, replies $\boldsymbol{F}$ Passariello. First of all what D Casian says can be true for lower limbs DVT, but is undoubtedly false for DVT-PE, as thrombosis can be everywhere and often the source of PE cannot be found, he argues. A consultation for a suspected DVT often is not a very urgent examination. People can wait one day or more for the exam, according to the availability of the specialist (for instance not at work now or in holidays). Try to apply this unavailability to a suspected PE, which can be a very critical disease (must enter or not ICU or coronary unit? must start a systemic thrombolysis ?). Time is a very high cost. $F$ Passariello thinks that an always-available simple CUS can give a much better service than an inconstant DUS service on demand. Finally, he suspects also that D Casian's false negative rate is much greater for DVT-PE than for DVT only. Secondly though he is a Vascular Surgeon, he doesn't think that Vascular Surgeons are the best professional profile to put an indication to DUS for VTE (DVT-PE) patients. Generally Vascular Surgeons are not involved in the use of scores, CUS, DUS, D-Dimer, sP-selectin and so on. The danger is that the indication could be given following common opinions and not what is reported in the literature and the guidelines. VTE patients show a clinical presentation in a range from life-threatening cases (cardiac arrest, acute respiratory failure, hemopthysis) to no relevance ones, with only a few not important symptoms or no one at all.

A Pieri comes into the discussion arguing with the followings: $P E$ is normally present (always) when one sees a DVT at any location (Angio CT scan always reveals small PE). Only clinically relevant $P E$ needs hospitalization and rarely fibrinolysys. Fatal PE is fatal! Otherwise full dose subcutaneous Heparin treatment is enough, waiting for a "second degree" diagnosis. We only need to make a "correct" Color Duplex diagnosis. CUS is not a good diagnosis because Doppler investigation is not included and because upper limbs (subclavian DVTs) and distal DVTs are not considered. Distal DVT are often the marker of PE (residual DVT after embolisation). DUS investigation of abdomen can reveal iliac, renal, ovarian, etc. locations of DVT, CUS and probability scores would not be allowed, in his mind, in a great Hospital. CUS could even be DANGEROUS in cases of proximal floating DVT (femoral-iliac)! We have only to decide when a CT scan is needed and when to investigate patients for cancer !! DDimer false positive (trauma or surgical patients) give no value to this kind of investigation that must be avoided (obviously in his mind). Only negative D-Dimer tests are useful because they can exclude a recent DVT. By the same ultrasound device we can investigate the 
heart to visualize cardiac chambers, pleural or pericardial effusions. Clinically relevant $P E$ can be diagnosed in few minutes. Color Doppler investigation can also offer alternative diagnosis.

A Puskas agrees with A Pieri's point of view regarding the better specificity and sensitivity of DUS and the potential risk of "aggressive" CUS as a cause of thrombus mobilization. F Passariello argues with the contrary: great hospitals SHOULD use scores and CUS, while avoiding them causes waste of resources. D Casian exemplifies different clinical scenarios (1. No symptoms of DVT + No symptoms of PE, 2. No symptoms of DVT + Symptomatic stable or unstable PE, 3. Symptomatic DVT + No symptoms of PE, 4. Symptomatic DVT + Symptomatic $P E$ ). These examples have as starting point mainly an already done diagnosis, while we are instead searching for a diagnosis, replies $\boldsymbol{F}$ Passariello. D Casian underlines that these clinical scenarios raise two questions: no routine screening for PE in asymptomatic patients and not to look for source of embolism in case of symptomatic PE and asymptomatic legs because patients in any case will be anticoagulated. In Scenario $n r 4 D$ Casian's opinion is that confirmation of PE is required only if patient is hemodynamically unstable, because it will influence the decision to start the thrombolysis. In scenario $\mathrm{nr} 1$ the investigation could be indicated only in high risk group. This could be achieved according to Caprini score, replies A Puskas. In scenarios nr 2 and 3 the imaging method will be selected to confirm DVT (DUS) or PE (CT angio) argues D Casian. C Franceschi agrees with D Casian's rational algorithm.

BB Lee's opinion is that better to do PE study BEFORE one starts the anticoagulation based on pragmatic view because de novo $P E$ development despite adequate anticoagulation is one of the indication for IVC filter placement. He also states that PE is NOT one shot episode. So anyone with PE, either hemodynamically stable or not, should have an assessment of 'current' PE status so that it will provide the BASELINE down the road for the future management, he argues. It is especially true for the DVT which is infrequently treated by the patient himself with a premature abandonment after a minimum due period. So proper assessment of PE with CTA at the beginning will provide a huge dividend with much reduced risk of a unique condition known as chronic thromboembolism (CTE) together with CTEPH, which is presumed to be due to recurrent pulmonary embolism-he comments. $D$ Casian in his reply depicts the reality in Moldova: even in big hospitals the trombolysis is not available at all; actually for more than 5 years they have no any thrombolytic agents in their country; they have only one interventional radiology suite (not available 24/24) in their country; zero cava-filters were implanted during the last 5 years; CT-angiography is performed only occasionally due to high cost and low availability (just in private centers) - in his hospital (University Hospital) there was 1 case during the last 10 years.

A Pieri says that indication of Cava filter is extremely rare nowadays. He states also that CUS is obviously included in DUS and that the first approach to DVT is heparin, then one can investigate for PE. F Passariello reminds the details of CUS and DUS (already outlined above in the text) and states that CUS is a separate exam and not a DUS. The latter of course includes compression of veins (everywhere and not only 2 points), but DUS does not consider venous compression as a step of a clinical algorithm. Thus though it uses compressions DUS does not include CUS. M Schul says that overutilization of CUS/DUS will be present until definitive guidelines will be established for DVT diagnosis and assessment. In his opinion CTA for PE investigation should be ordered on clinical basis alone. $\boldsymbol{C}$ Franceschi writes about plantar veins thrombosis as a possibility and this should be checked because it is rare but exists and can be efficiently assessed by DUS ${ }^{42}$. He also states that most of the time a false positive diagnosis regards the peroneal vein, because it is not easy to compress completely just with the probe. In that case, C Franceschi suggests an interesting and practical tip: a simultaneous additional compression with the free hand and/or the leg elevation more than $45 \%$ provides a complete vein collapse, when the vein is free of clot/thrombus.

A Puskas calls for attention regarding the position of the patient during DUS examination which is crucial. For instance for the popliteal vein and below the best position is not in the ventral decubitus, but sitting at the free margin of the examination table, while the foot does not touch the floor. The examination is done from behind the knee and at the medial and lateral margin of the calf, internal malleolus and at the plantar level. In this way the calf is relaxed and the compression is easy to do with the probe and simultaneously with the free hand. Standing is also mandatory because sometime it is really a challenge to discriminate between chronic thrombus with partial reopening and acute recurrence on chronic thrombosis or in case of incomplete acute thrombus. Standing is the only way to detect reflux with provocative manoeuvres in diastole (Paraná). In the case of chronic residual thrombus the color filling is within the remnant/ residual thrombus which is "pierced" by the recanalization (A Puskas calles it the "cavernous" type), whilst in an acute incomplete occlusion the color flow is marginal and visible mainly in systole of the calf (Paraná). It is also true that recanalization can be "marginal" or "parietal" as he called in one of his paper but in this case the diastolic reflux is present at Paraná manoeuvre.

A Pieri argues again in favor of DUS which is the method of choice in his opinion. It is very fast and safe to rule out DVT by complete DUS, he says. DUS also permit a possible differential diagnosis in approximately $90 \%$ of the cases with no need to access $3^{\text {rd }}$ degree investigations which, in Italy at least, are much more expensive. F Lurie summarizes the discussion until this point as follows: this discussion confirms that the utility of any imaging technique depends on the practice settings, access to treatment options and local standard of care. For example, if thrombolysis is not available and is not a local standard of care all ilio-femoral DVTs will be treated the same as femoro-popliteal DVTs and the value of imaging ileo-caval segment is questionable. In other settings, when the catheter-directed thrombolysis is a standard of care for ilio-femoreal DVT, the ileo-caval imaging is absolutely necessary. G Stansby argues that CUS is the minimum and should be widely available and is very good for confirming proximal DVT. A venous specialist to see and investigate all potential DVTs with DUS would be ideal but that isn't the real world in most places, he writes.

BB Lee emphasizes again that all the PE deserve to be clarified together with DVT as a part of VTE investigation when clinically suspicious enough to mandate further laboratory test. Whether the PE is small or large, and/or hemodynamically stable or unstable, the PE is a PE so that it has to be verified with appropriate measurement. Many institutes incorporated CTPA as a first-line imaging study in cases of 'suspected' acute PE. Traditional V/Q scintigraphy is no longer first option unless motion artifact of poor right heart function limits the quality of CTA or CTA is contraindicated due to the allergic reaction to radiographic contrast. The sensitivity and specificity of CTPA for diagnosis of acute PE have been reported to range from $53 \%$ to $100 \%$ and $67 \%$ to $100 \%$ respectively varying on the basis of patient selection, extent of thrombus, area of the vasculature imaged, interpretation criteria, and experience of the reader, he states. He also adds that modern MDCT or multi-detector CT (MDCT) scanners are an entirely different ball game in comparison to single-slice CT scanners we all are familiar with.

The new one is able to deliver the images with sufficient resolution to delineate intraluminal filling defects with a sharp interface with intravascular contrast material. In chronic PE, for instance, it delivers clear evidence of recanalization, webs or flaps, and partial filling defects that form obtuse angles with the vessel wall. So common impressions on the CTA are often for old CT scanners while the new ones are quite different to provide proper differentiation of this unique 
pathologic condition, whether acute or chronic, causing both partial and complete intraluminal filling defects in a variety of condition to assess properly, he argues. Regarding the 'asymptomatic' PE, found incidentally during the investigation of DVT or rather VTE he states as follows: I don't know whether this 'asymptomatic' PE should be considered same as so called 'unsuspected' PE but current guidelines from the American College of Chest Physicians clearly recommend same treatment like symptomatic $P E$ in the absence of convincing evidence that anticoagulation can be safely withheld. Of course, I agree that the increasing use of CTA has led to the increased diagnosis of incidental 'asymptomatic' PE and/or small sub segmental PE (SSPE) which is not clear for its clinical relevance and the optimal therapy for SSPE is still on debating. Indeed, a single sub segmental defect probably does not have the same clinical relevance as a single segmental or lobar PE or multiple sub segmental PE. But, most experts agree such SSPE should be treated like symptomatic or large lobar $P E$ especially when the risk factors for VTE persist, and there is concomitant DVT. Of course, the duration of anticoagulation in such cases should be individualized. Such sophisticated/advanced strategy would have not been possible without advanced MDCT and we would have not been able to reach this far in terms of future long term outcome.

He also says that in some countries, like China, the DUS is more expensive than CTA. In US the examination is heavily affected by the insurance payment system so that doctors simply endorse the DVT investigation algorithm based on DUS with not much appreciation on the CUS though its value has been acknowledged, not inferior to DUS. Nevertheless, CUS has been known for limited utility for the detection of isolated iliac vein thrombosis -failure to identify such thrombi may prove fatal-, and less sensitive for the detection of isolated calf vein thrombosis in symptomatic patients. Also, non-compressibility limited to the common femoral vein often does not represent venous thrombosis in patients with extensive pelvic disease (e.g., neoplasm or radiation). Indeed, when clinical suspicion is low or when circumstances favor a falsely positive CUS, he has no doubt a more aggressive diagnostic approach is necessary.

In such case, alternative approaches such as MRV/CTV would be needed together with standard DUS to confirm or exclude the diagnosis in his opinion. In spite of such disadvantages, many European physicians seem to use CUS as the initial diagnostic test for patients with symptoms or signs that suggest DVT based on well documented safety, accuracy and availability. And further they rely on negative CUS finding alone for decision to withhold anticoagulants on the day of presentation and again 5 to 7 days later. Indeed, combining D-dimer tests or clinical probability-low or moderate - with a single negative CUS exam seems to be safe for management of 'suspected' DVT among the patients. But in the U.S., CUS has been faded away from active leading role.

G Stansby replies with the statement that if the patient has a DVT and will be anticoagulated anyway a CTPA is not indicated if there is no clinical suspicion of PE. As a conclusion BB Lee emphasizes again that he does agree categorically with 'NO routine screening for PE on every DVT. But, when in doubt with a high suspicion, he would not hesitate to go beyond basic assessments of PE including Wells PE score and proceed with further solid/substantial assessment including the CTPA if feasible. That is my first message on the CTA involved to VTE assessment, he says. Second, he would like to caution on the statement: "Confirmation of PE is required only if patient is hemodynamically unstable". More we learned on the pathogenesis of CTEPH through the last decade, more we were surprised with its intimate relationship with chronic thromboembolism (CTE). So we know now clearly that CTEPH is presumed to be due to recurrent pulmonary embolism until proven otherwise. So he strongly advocates all the PEs, either hemodynamically stable or unstable, deserved to have proper assessment of the status of 'proven/confirmed' PE as the BASELINE evaluation for the future management. Indeed, appropriate assessment of the PE with CTA at the beginning will guide/ provide proper strategy to tackle with such unique condition of CTE/CTEPH. He strongly advocates the CTA on the management point of view as well but, ONLY when one have enough knowledge to imply its findings to proper treatment strategy to retrieve its benefit in maximum. Casual practice/commitment to CTA/CTPA without appropriate disposition should be discouraged.

In PE diagnostics CT angio has an edge over MR angio (M Patel, $N$ Labropoulos). M Patel raises the problem of transportation of PE suspect patient. Transportation even within the hospital has been shown to be a risk factor, he continues. In fact many accidents occur while patients are transported. Unstable patients have to be diagnosed with whatever best the hospital has. Unfortunately treating such a patient in a place where diagnostic facilities are not optimum can lead to litigation. So transportation is required for diagnosis and be as safe as possible, he concludes. $\boldsymbol{G}$ Stansby agrees with him and says that for suspected PE patients usually it is best to start heparin immediately. If investigations (CTPA) are delayed then at least they are already covered by therapy. Another point for those without CTPA, V/Q spect is very promising as an alternative, he adds.

As regard to treatment options and for more aggressive thrombolysis indications $\boldsymbol{B B}$ Lee states that their standing policy is 'thrombolysis of any term, preferably PMT (percutaneous mechanical thrombectomy) unless otherwise indicated/contraindicated'. Even for the thrombus more than two weeks old, we do bend backward to try whenever the patient should give proper consent, he adds. Indeed, the 'limited' experience shows such flexible implication even to chronic thrombus is worthy with consideration of stenting, he continues. His personal feeling is that 'still better than nothing' as a believer of Tony Comerota's crusade despite persistently adamant ACP guideline. Regarding subsegmental PE (SSPE) he writes that the cumulative risks for VTE as well as death are in same ranges among the SSPE and proximal PE groups so that all PE, including SSPE, should be considered for anticoagulant therapy since its benefits outweigh the risks of complications. Hence, most of leading institutes advocate anticoagulant therapy to the symptomatic SSPE as long as there is no absolute contraindication or high risk factor of bleeding. We do have clear evidence to verify the effectiveness and safety of anticoagulation therapy versus no intervention on SSPE based on randomized controlled trial, he adds. Nevertheless, he mentions, that advanced CTPA revolutionized the diagnosis of PE including the smaller emboli in subsegmental pulmonary arteries so that it is able to diagnose relatively unimportant emboli for which the risks of anticoagulant therapy may not be warranted under the claim for persistent risk factors for recurrent VTE!

In his practice M Patel has very strict indications for use of an IVC filter. He uses it only when there is an absolute contraindication for anticoagulation. CTPA in India costs about USD 150-200 on a 64 or 128 slice CT scanner. We have to get maximum yield out of the test we order, he claims. For now, CTEPH will be a diagnosis based on high index of clinical suspicion and the scoring systems we are discussing on this board will throw more light on what should become standard procedure, he concludes. BB Lee comments that if the situation is serious enough to start the anticoagulation, it would be better to do PE study BEFORE starting the anticoagulation based on pragmatic view, because one of major indication for IVC filter placement is "de novo" $P E$ development despite adequate anticoagulation! However, many, though mostly anecdotal, PEs were already there before one starts the anticoagulation so that belatedly found PE does NOT always means "de novo" as the outcome of anticoagulant failure to justify IVC filter, which was designed to reduce only the risk of 'fatal' PE and NOT for all the minor blood clots which continue to travel, he writes.

F Passariello introduces the discussion about a recently published clinical case of fatal endovascular thrombectomy ${ }^{43} . \mathbf{N}$ 
Labropoulos comments that there are several methods for performing Pharmaco-Mechanical-Thrombectomy (PMT) in patients with PE and the presented case was not a real mechanical one. M Simka adds that this case was not a mechano-chemical but only chemical thrombectomy with the addition of an old-fashioned mechanical component. Currently, a real mechanical thrombectomy can be used for the treatment of critically-ill PE patients as a life-saving therapy. In addition, patients with sub-massive PE can also be managed in this way, but at the moment it is not a routine procedure in such cases - perhaps with more evidence it will be used more often, he argues. F Passariello completely agrees with M Simka about the old fashioned method used in the presented case. In addition, the rotational manoeuvre in the video lasts only a while, has no downstream protection and is not at first sight able to insure a result, comments. Actually PMT is not considered yet a primary tool in a very severe PE and guidelines should plan a more aggressive intervention when the clinical case requires it, he concludes.

\section{Algorithms}

The British National Institute for Health and Care Excellence $^{44}$ (NICE) developed two important algorithms, the first for DVT, the latter for PE. Here we will take them just as an example of all the other existing algorhitms. Both are drawn for the ER or for a structure able to insure very quick evaluations. This is an important point, because the Wells DVT score is used in an ER environment, other than ambulatorial.

Both algorithms consider a short term(4h) ultrasound availability. DUS is not cited at all, while the term proximal ultrasound appears as a substitute for CUS.

In this regard several legitimate questions arise:

-is the proximal ultrasound term an analogous for a proximal CUS, i.e. performed only at the SFJ ?

-Is there an evidence-based medicine (EBM) available reference for the proximal CUS ?

-Is the proximal CUS documented to have the same sensitivity of a complete CUS.

-In addition, when time needed to get an ultrasound investigation is $>4 \mathrm{~h}$ and reaches 1 day or more, why not having a complete DUS instead of a CUS ? The same question holds if a repeated examination is needed one week later.

The reported flow charts are clear: always perform a score, there is no doubt about. However, scores are different for DVT and PE, though they are both Wells score. Why Wells PE and not Geneva for PE ? Wells PE is more operator dependent, because one of the items is subjective, exactly where the score asks if there is an alternative diagnosis. Geneva instead is operator independent and more repeatable. Interestingly, an interim treatment with heparin is always planned when investigations are not soon available. This feature means that NICE algorithms can be regarded as mixed ones, i.e. diagnostic + therapeutic. It seems that for the more urgent and life-threatening cases the diagnosis must be mixed with the therapy.

The PE NICE algorithm plans a reduced time threshold of $1 \mathrm{~h}$ instead of $4 \mathrm{~h}$ in order to choose between ultrasound and D-Dimer and introduces also CTA between diagnostic tools. In addition, the PE flow chart is graphically redundant, thus it could be simplified becoming more readable, but leaving the content unchanged.

Another last observation deals with the choice between these algorithms and the other ones we saw for the outpatients. In simple words, outpatients flow-charts should be modified to allow exiting with the urgent need of shortening the procedures, i.e. sending the patient from the ambulatory to the hospital or to the ER.

\section{Conclusions}

At the end of the discussion, the Moderator $\mathbf{F}$ Passariello tries to resume the still unclear and open points.

There are two great approaches in VTE diagnosis:

A. The imaging techniques are constituted in general by an anatomic and hemodynamic point of view, the detection of thrombosis being based definitely on its visibility In this group also the pressure evaluation is included (an endo-venous invasive measurement as well as a non-invasive one), as it deals with a mixed anatomical/ hemodynamic context. Examples in this group are: CUS, Duplex, phlebography, pulmonary angiography, CTA, MRA, V/Q scintigraphy, endo-venous catheter pressure measurement, non-invasive pressure measurements, etc.

B. The statistical tools include the scores and the biomarkers assays and clarify if thrombosis exists or not, but do not say nothing about its anatomical localization Examples are Wells (DVT and PE) scores, revised Geneva, PERC rule, D-Dimer, sP-selectin.

The contra-opposition is evident. The fans of the imaging methods will look with suspect at scores and biomarkers, asking instead "where" thrombosis is located and which are its features. On the other side, the fans of the statistical tools will criticise the negative findings of the imaging techniques, because very often thrombosis hides itself and it is very difficult to find it: if you are not able to see thrombosis, it doesn't mean that it is not there.

In addition, there are two great styles in VTE diagnosis:

a. the expert opinion which is more near to the clinicians' mind, with all the criticism which can be moved to it. Clinical judgements like the "Gestalt" associated to the PERC rule are included in this group. Also DUS (with the clinical and instrumental intuition associated to the use of 
the "III Chakra Eye" constituted by the ultrasound probe) gives the opportunity of expressing an expert opinion.

b. The standard forms and procedures of the algorithms pertain instead to another style, very near to EBM Medicine, which tries to limit as much as possible the role of the expert opinion in medical procedures.

\section{Acknowledgements}

The VTE_Risk Vasculab Debate was organized by the Vasculab List as an Online Debate. We thank all the participants for their contribute and especially for their active role: Pier Luigi Antignani, Benedetto Bernardo, Malika Boucelma, Victor Manuel Canata, Dumitru Casian, Tom Eaton, Khalil Fattahi, Francesco Ferrara, Carmine Finelli, Claude Franceschi, SB Gogia, Mitchel P. Goldman, Kuntelia, Nicos Labropoulos, Christopher R Lattimer, Zaza Lazarashvili, Byung Boong Lee, Fedor Lurie, Wademar Lech Olszewski, John Opie, Fausto Passariello, Malay Patel, Giampiero Peruzzi, Alessandro Pieri, Norma Poli, Attila Puskas, Cestmir Recek, Steve Szarka, Heraldo Schlup, Marlin Schul, Marian Simka, Gerard Stansby, Roy Varghese. efforts.

Finally, we thank Mrs. Iolanda Palma for her organizational

\section{References}

1) Haeger K. Problems of acute deep venous thrombosis. II. Mobilization and discharge of the patient. Angiology 1969;20:280-6.

2) Hull R, Hirsh J, Sackett DL, Stoddart G. Cost effectiveness of clinical diagnosis, venography, and non-invasive testing in patients with symptomatic deep-vein thrombosis. N Engl J Med 1981;304:1561-7. DOI: 10.1056/NEJM198106253042602

3) Oudega R, Moons KG, Hoes AW. Limited value of patient history and physical examination in diagnosing deep vein thrombosis in primary care. Fam Pract 2005;22:86-91. http:// fampra.oxfordjournals.org/content/22/1/86.long DOI: 10.1093/fampra/ $\underline{\mathrm{cmh} 718}$

4) Anand SS, Wells PS, Hunt D, Brill-Edwards P, Cook D, Ginsberg JS. Does this patient have deep vein thrombosis ? JAMA 1998;279:1094-9. http://jama.jamanetwork.com/article.aspx? articleid $=187418$

5) Wells PS, Anderson DR, Bormanis $J$ et al. Value of assessment of pretest probability of deep-vein thrombosis in clinical management. Lancet 1997;350:1795-1798.

6) Wells PS, Anderson DR, Rodger M, Forgie M, Kearon C, Dreyer J. et al. Evaluation of D-Dimer in the Diagnosis of Suspected Deep-Vein Thrombosis. N Engl J Med 2003; 349:1227-1235September 25, 2003 DOI: 10.1056/NEJMoa023153

7) Wells PS, Anderson DR, Rodger M, Ginsberg JS, Kearon C, Gent $\mathrm{M}$ et al. Derivation of a simple clinical model to categorize patients probability of pulmonary embolism: increasing the models utility with the SimpliRED D-dimer. ThrombHaemost 2000;83:416.420.

8) Le Gal G, Righini M, Roy PM, Sanchez O, Aujesky $\mathrm{D}$, Bounameaux $\mathrm{H}$ et al. Prediction of pulmonary embolism in the emergency department: the revised Geneva score. Ann Intern Med 2006; 144:165-171.

9) Penaloza A, Verschuren F, Dambrine S, Zech F, Thys F, Roy PM. Performance of the Pulmonary Embolism Rule-out Criteria (the PERC rule) combined with low clinical probability in high prevalence population. Thrombosis Research 129 (2012) e189\#e193. Available at the address http://www.thrombosisresearch.com/article/ S0049-3848(12)00064-3/abstract at the date of Feb 8, 2017. 2012 DOI: 10.1016/j.thromres.2012.02.016

10) Schwartz DT. Problems With PERC. Available at the address http://www.annemergmed.com/article/ S0196-0644(10)00487-7/fulltext at the date of Feb 8, 2017. DOI: 10.1016/j.annemergmed.2010.04.032

11) Kline JA. More on the PERC Rule. Available at the address http://www.annemergmed.com/article/ S0196-0644(11)01441-7/fulltext at the date of Feb 8, 2017. DOI: 10.1016/j.annemergmed.2011.07.041

12) Hugli O, Righini M, Le Gal G, Roy PM, Sanchez O, Verschuren F, Meyer G, Bounameaux H, Aujesky D. The pulmonary embolism rule-out criteria (PERC) rule does not safely exclude pulmonary embolism. J Thromb Haemost 2011; 9: 300-4. Available at the address http://onlinelibrary.wiley.com/doi/10.1111/ j.1538-7836.2010.04147.x/epdf at the date of Feb 8, 2017. DOI: 10.1111/j.1538-7836.2010.04147.x

13) Caprini JA. Risk assessment as a guide to thrombosis prophylaxis. Curr Opin Pulm Med. 2010 Sep;16(5):448-52. DOI: 10.1097/MCP.0b013e32833c3d3e.

14) Barbar S, et al. A risk assessment model for the identification of hospitalized medical patients at risk for venous thromboembolism: the Padua Prediction Score. J Thromb Haemost 2010;8:2450-7.

15) Aujesky D, Obrosky DS, Stone RA, Auble TE, Perrier A, Cornuz J, Roy PM, Fine MJ. Derivation and validation of a prognostic model for pulmonary embolism. Am J Respir Crit Care Med. 2005 Oct $15 ; 172(8)$

16) Pisters R, Lane DA, Nieuwlaat R, de Vos CB, Crijns HJ, Lip GY. A novel user-friendly score (HAS-BLED) to assess 1-year risk of major bleeding in patients with atrial fibrillation: the Euro Heart Survey. Chest. 2010 Nov;138(5):1093-100

17) Tiganas D. Diagnostic value of the clinical probability score of deep venous thrombosis in the elderly. (French) La revue de médecine interne 26 (2005) 93-937. DOI: 10.1016/ j.revmed.2005.08.010

18) The VLAB Geneva Form. Available at the address http:// www.vasculab.it/VTE/Geneva.htm at the date of Feb 8, 2017.

19) Mousa AM, Broce M, Gill G, Kali M, Yacoub M, AbuRahma AF. Appropriate Use of d-Dimer Testing Can Minimize Over-Utilization of Venous Duplex Ultrasound in a Contemporary High-Volume Hospital. Ann Vasc Surg 2015;29:311-317. DOI: 10.1016/j.avsg.2014.07.032

20) Thachil J, Fitzmaurice DA, Toh CH. Appropriate Use of Ddimer in Hospital Patients. The American Journal of Medicine (2010) 123, 17-1.

21) Brotman DJ, Segal JB, Jani JT, et al. Limitations of D-dimer testing in unselected inpatients with suspected venous thromboembolism. Am J Med. 2003;114:276-282. DOI: 10.1016/ S0002-9343(02)01520-6.

22) Larsen TB, Stoffersen E, Christensen CS, et al. Validity of D-dimer tests in the diagnosis of deep vein thrombosis: a prospective comparative study of three quantitative assays. J Intern Med. 2002;252:36-40. 
23) Pulivarthi S, Gurram MK. Effectiveness of D-Dimer as a Screening Test for Venous Thromboembolism: An Update. N Am J Med Sci. 2014 Oct; 6(10): 491\#499. DOI: 10.4103/1947-2714.143278

24) Vandy FC, Stabler C, Eliassen AM, Hawley AE, Guire KE, Myers DD, Henke PH and Wakefield TW. Soluble P-selectin for the diagnosis of lower extremity deep venous thrombosis. JVS. 2013;April:117-25. DOI: 10.1016/j.jvsv.2012.09.001

25) Gremmel T, Ay C, Seidinger D, Pabinger I, Panzer S, Koppensteiner R. Soluble p-selectin, D-dimer, and high-sensitivity Creactive protein after acute deep vein thrombosis of the lower limb. JVS. 2011;54(6 Supplement): 48S-55S. DOI: 10.1016/j.jvs.2011.05.097

26) Passariello F. La cascata dell'ossigeno al letto del paziente. [Bedside oxygen cascade]. Seminar. Napoli, Dec 16, 2014. DOI: 10.24019/2014.BO2C.

27) Passariello F. La cascata dell'ossigeno al letto del paziente. [Bedside oxygen cascade]. Presentation to the Meeting Insufficienza respiratoria acuta su cronica. Nuovi modelli assistenziali dall'ospedale al territorio. Napoli, Jun 5, 2015.

28) Passariello F, Angelini V, Bellinfante E, Curcio I, Helzel V. Stima del rapporto Ventilazione-Perfusione nel paziente critico con insufficienza respiratoria. [Estimate of the Ventilation-Perfusion ratio in the critical patient with respiratory failure]. Presentation to the X National Meeting of SIMEU (Italian Society of Emergency and Urgence Medicine. Napoli, Nov 18-20, 2016.

29) Cogo, A., Lensing, A.W.A., Koopman, M.W.M., Piovella, F., Siragusa, S., Wells, P.S., Villalta, S., (...), Prandoni, P. Compression ultrasonography for diagnostic management of patients with clinically suspected deep vein thrombosis: Prospective cohort study . (1998) British Medical Journal, 316 (7124), pp. 17-20.

30) Bernardi E et al. Extended Versus Rapid Analysis of the Leg Vein System: a Multicenter Ultrasound Study (The ERASMUS Study) - An Interim Analysis. Pathophysiol Haemost Thromb 2003; 33 (5-6), pp. 314-318.

31) Bernardi et al. Serial 2-point ultrasonography plus D-dimer vs whole-leg color-coded Doppler ultrasonography for diagnosing suspected symptomatic deep vein thrombosis: A randomized controlled trial. JAMA 2008; 300 (14):1653-1659.

32) VLAB-CUS form (Italian). Available at the address http:// www.vasculab.it/VTE/CUS form.htm at the date of Feb 8, 2017.

33) VLAB-CUS form (English). Available at the address http:// www.vasculab.it/VTE/CUS form en.htm at the date of Feb 8, 2017.
34) Worsley DF, Atavi A. Comprehensive analysis of the results of the PIOPED Study. J Nucl Med. 1995;36:2380-2387.

35) The PIOPED Investigators. Value of the Ventilation/ Perfusion Scan in Acute Pulmonary Embolism JAMA 1990;263:2753-2759.

36) He J, Fang W, Lv B, He JG, Xiong CM, Liu $\mathrm{ZH}$, He ZX. Diagnosis of chronic thromboembolic pulmonary hypertension: comparison of ventilation/perfusion scanning and multidetector computed tomography pulmonary angiography with pulmonary angiography. Nucl Med Commun. 2012 May;33(5):459-63. https://www.ncbi.nlm.nih.gov/pubmed/22262242 at the date of Feb 8, 2017.DOI: 10.1097/MNM.0b013e32835085d9.

37) Hutchinson BD, Navin P, Marom EM, Truong MT, Bruzzi JF. Overdiagnosis of Pulmonary Embolism by Pulmonary CT Angiography. AJR Am J Roentgenol. 2015 Aug;205(2):271-7. DOI: 10.2214/AJR.14.13938.

38) Granovetter M. CTPA use reignites debate on overdiagnosis. The lancet Respiratory Medicine. 2013;1(6):436. DOI: $\underline{10.1016 /}$ S2213-2600(13)70168-1.

39) Hochhegger B, Ley-Zaporozhan J, Marchiori E, Irion K, Soares Souza A, Jr, Moreira J, Kauczor H U, and Ley S. Magnetic resonance imaging findings in acute pulmonary embolism. Br J Radiol. 2011 Mar; 84(999): 282\#287. DOI: 10.1259/bjr/26121475

40) Revel MP, Sanchez O, Couchon S, Planquette B, Hernigou A, Niarra R, Meyer G, Chatellier G. Diagnostic accuracy of magnetic resonance imaging for an acute pulmonary embolism: results of the 'IRM-EP' study. J Thromb Haemost. 2012 May;10(5):743-50. DOI: 10.1111/j.1538-7836.2012.04652.x.

41) Schiebler ML, Nagle SK, François CJ, Repplinger MD, Hamedani AG, Vigen KK, Yarlagadda R, Grist TM, Reeder SB. Effectiveness of MR angiography for the primary diagnosis of acute pulmonary embolism: clinical outcomes at 3 months and 1 year. J Magn Reson Imaging. 2013 Oct;38(4):914-25. DOI: 10.1002/jmri.24057. Epub 2013 Apr 1.

42) Franceschi C. Thrombophlebite des veines plantaires. Actualités Vasculaires Internationales. 1997;47:29-39.

43) Oguzhan A. Percutaneous Rotational Pulmonary Thrombectomy. Images in Clinical Medicine. N Engl J Med 374:e1, January 7, 2016. Available at the address http://www.nejm.org/doi/ full/10.1056/NEJMicm1415847?query=emergency-medicine at the date of Feb 08, 2017. DOI: 10.1056/NEJMicm1415847.

44) The National Institute for Health and Care Excellence (NICE). Venous thromboembolic diseases: diagnosis, management and thrombophilia testing. https://www.nice.org.uk/guidance/cg144 